\title{
¿Qué comparar cuando se comparan las desigualdades en los sistemas educativos? Más allá de las desigualdades escolares, la reproducción social
}

\author{
Valdivia Yábar, Silvia Verónica \\ Universidad Nacional de Arequipa, Arequipa, Perú \\ siveroval@gmail.com
}

\section{Resumen}

Mientras que el desarrollo de las comparaciones internacionales alimenta cada vez más los debates y las políticas en materia de educación, la cuestión de las bases sobre las cuales se fundamentan estas comparaciones está raramente planteada. La dimensión académica - el nivel de logro de los estudiantes y las desigualdades relacionadas- está de hecho privilegiada, aunque para la mayoría de los países, en última instancia, el objetivo es la inserción de las generaciones más jóvenes en la vida social y económica. Esta ponencia defiende la idea de que una práctica de la evaluación de los sistemas educativos no puede dejar de tener en cuenta los procesos de inserción de los jóvenes, así como la fuerza de inmovilidad / movilidad entre generaciones. Ciertamente, al hacerlo, se sitúa más en la perspectiva de una evaluación del rol de la escuela en la sociedad que en una estimación del impacto de tal o cual modalidad de su funcionamiento. Y esto puede ser discutido, ya que conduce a explicar lo que, en las políticas educativas, constituye el objetivo final.

\section{Abstract}

While educational debates and policies do rely more and more often upon international comparisons, the question of the basis on which these comparisons may be made is seldom raised explicitly. The academic dimension -the level of pupils attainment and the correlated inequalities- is de facto put forward, in spite of the fact that in most countries, what is at stake is generally to integrate the young generations in social and economic life. This paper maintains that the evaluation of educational systems should not omit to take into account the process of access to adulthood, and also the strength of social mobility (or immobility) between generations. So doing, one adopts more the perspective of assessing the part played by school within society, rather than estimating the impact of such and such school components. That may be debated, while it leads to make explicit what is aimed at through educational policies.

Palabras clave: comparaciones internacionales, desigualdades sociales, rendimiento de los diplomas, inserción de los jóvenes, movilidad social, reproducción social.

Keywords: international comparisons, social inequalities, returns of diploma, access to jobs (young people), social mobility, social reproduction.

\section{INTRODUCCIÓN}

Las comparaciones de los sistemas educativos se han multiplicado, bajo dos modalidades: los enfoques cualitativos en un número limitado de países o bien vastas encuestas en un gran número de países, para los cuales los datos comparables están disponibles. En el primer caso, se hizo un análisis detallado de los modos de funcionamiento, situándolos en su contexto histórico, social, sin necesariamente saber si los aspectos seleccionados tienen alguna pertinencia externa, es decir, si afectan a los estudiantes, sus logros o sus actitudes. De otro lado, es difícil demostrar, frente a los logros desiguales, que este o aquel aspecto de los sistemas sea la causa de lo adquirido. Esto es cierto, ya que se limita a menudo a los enfoques correlacionales y, en este sentido, los modelos multivariados, que evalúan los efectos netos de tal o cual parámetro y controlan un gran número de variables, no ofrecen una perspectiva significativamente mejor. 
Además, la pregunta es raramente explícita sobre qué conviene comparar y los puntos de vista pueden diferir según las disciplinas académicas de los investigadores. Para los especialistas de ciencias de la educación, parecen obvios los logros y las desigualdades, que marcan a los estudiantes, más raramente las actitudes son las dimensiones a privilegiar, para comparar los modos de funcionamiento de los sistemas. Modos de funcionamiento, en los cuales los economistas se centran para evaluar la eficiencia. Para los sociólogos, es la reproducción social, que se juega, lo que importa y, en este sentido, solo interesa lo que produce la escuela, lo que augura de la producción (y reproducción) de desigualdades.

Esta reproducción se juega por dos procesos diferentes, como lo muestran los análisis de Kerckoff (1995), que distingue un proceso de socialización y un proceso de asignación de empleos (basado en la formación recibida). De hecho, la sociología de la movilidad social ha adoptado esta doble perspectiva -con el triángulo omnipresente OED, donde la reproducción entre $\mathrm{O}$ - el origen social - y $D$ - el destino social- se desarrolla por la relación O-E (relaciones entre el origen social y la educación recibida), luego por la relación E-D (relaciones entre educación y empleo). Pero, los sociólogos de la educación, Bourdieu y Passeron han privilegiado la vía de la socialización en relación con la vía de asignación, sin duda porque la inserción aparecía entonces no problemática. Sin embargo, una serie de trabajos comparativos muestra que el segundo proceso varía más de un país a otro que las desigualdades sociales de logros, lo que debería a priori volver más heurísticas las comparaciones.

\section{CÓMO LA REPRODUCCIÓN COMIENZA EN LA ESCUELA}

Las desigualdades sociales de los logros entre los estudiantes de 15 años son bien conocidas desde las encuestas PISA. En los trabajos recientes (Amarante, 2015), se ha cruzado el nivel de estas desigualdades escolares y el índice de Gini; y la correlación entre estas dos series, en las muestras de países de América Latina, es alta y significativa. En estos casos, el modelo de reproducción parece validado, ya que las desigualdades escolares tienen una amplitud comparable con las desigualdades sociales.

Las desigualdades entre los estudiantes dependen también del nivel de segregación social y escolar de las instituciones, que resulta de la manera en la que se agrupan los estudiantes, que a su vez depende de las políticas escolares y del nivel de segregación espacial de las desigualdades sociales. Es relevante distinguir dos parámetros sintéticos, como lo hacen Demeuse y Baye (2008). Primero, mide la segregación del público escolar a los 15 años desde varios ángulos: escolares, sociales, origen lingüístico. El otro mide el carácter segregativo de las estructuras escolares, que se evalúan al agregar los modos de agrupación de los estudiantes, la proporción de recursos privados, la elección de la escuela dejada a la iniciativa de los padres, la tasa de repetición. Estas dos variables están obviamente correlacionadas y la relación sería alta como se puede suponer. Pero, la segregación social y escolar entre las instituciones, que prevalece en los países, está asociada a fuertes desigualdades escolares. El desigual grado de segregación de los estudiantes aparece así como un parámetro importante de diferenciación de los sistemas educativos en materia de desigualdades sociales de logros. Esta segregación y las desigualdades, que están asociadas, pueden resultar de las políticas educativas, tales como las políticas de libre elección de la escuela. Al contrario, del lado de los sistemas menos desiguales, se encuentran los países donde la elección de la escuela está regulada, la descentralización está enmarcada y la participación privada es débil.

En suma, las diversas formas de organizar los estudios juegan un papel importante en la construcción de las desigualdades sociales del rendimiento escolar, y el conjunto de estos resultados es bien conocido. Pero tanto el investigador en educación como el sociólogo hacen dos observaciones. En primer lugar, la mayoría de los análisis se centran en la comparación de los logros (PISA), que es, por supuesto, justificado; pero esto no agota todos los propósitos de la acción educativa. Siempre se tiene como objetivo desarrollar en los estudiantes ciertas actitudes, ciertamente más difíciles de aprender, pero cuyas primeras exploraciones muestran que no parecen seguir exactamente la jerarquía de los logros, ya que no hay correlación, por ejemplo, entre los puntajes de las pruebas de conocimiento y los juicios más o menos positivos de los estudiantes sobre la escuela, el placer que tienen de ir allí o la calidad de sus relaciones con los profesores (Duru-Bellat, Mons y Bydanova, 2008).

Por otro lado, los logros no son «simplemente» tomados en cuenta porque la adquisición de conocimientos constituya un fin en sí, sino que suponen una inserción económica y social más o menos satisfactoria. En este caso, las desigualdades que marcan estos logros y diplomas serán el vector de la reproducción social de las posiciones, de los padres a los hijos. 


\section{EL INSTRUMENTO ESENCIAL DE LA REPRODUCCIÓN}

Sin embargo, hay pocos análisis comparativos que exploran empíricamente esta cuestión del papel que juegan las desigualdades escolares certificadas por los diplomas en la formación de las desigualdades sociales avaladas por la escuela. Es probable que algunas sociedades valoren más o menos los diplomas y desarrollen los sistemas de formación y/o de calificación alternativos a la escuela, lo que modulará la fuerza de la relación entre los diplomas escolares y las posiciones sociales posteriores (Shavit y Müller, 1998). Entonces, las comparaciones entre sistemas educativos deben ampliar su campo de análisis al proceso de inserción y a la forma en que los jóvenes rentabilizan sus diplomas.

Para hacer esto, se debe estudiar cómo el diploma se traduce en ingresos y en estatus social, lo que se denomina influencia del diploma (Dubet, Duru-Bellat y Vérétout, 2010). Se puede concebir que, la variación de la intensidad de las relaciones entre las desigualdades de logros escolares y las desigualdades sociales se explica por la incidencia desigual, según los países, de los logros y diplomas escolares en el futuro profesional de las personas. En este caso, el papel de la escuela en el proceso de reproducción social dependerá tanto si no más del valor socioeconómico de los títulos, que ofrece, como de las desigualdades sociales de obtención de dichos títulos. Además, una hipótesis sociológicamente probable es que cuando la influencia de los diplomas en las posiciones sociales es fuerte, habrá una competencia intensa entre los grupos sociales para asegurar la mejor posición posible a sus hijos y los grupos favorecidos tendrán por su posición más oportunidades de lograrla.

Para probar esta hipótesis, se construyó una variable denominada «influencia», que aborda el alcance de los beneficios económicos aportados por el diploma, específicamente por un diploma del nivel superior, que constituye el más discriminatorio cuando se trabaja en una muestra de países escolarizados en este nivel. La primera constatación: el «rendimiento» de los diplomas es desigual según los países. La segunda constatación: la influencia de los diplomas se correlaciona con la magnitud de las desigualdades de ingresos. La relación puede ser también en la dirección opuesta: cuando las desigualdades de salario son fuertes, es tanto más necesario justificarlas con un criterio considerado como legítimo y en las sociedades donde reina esto, que Goldthorpe (2014) llama una «educación basada en la meritocracia», los diplomas son el criterio ideal.

Conviene enfatizar que, una sociedad conforme al modelo meritocrático conjugaría una ausencia de desigualdades sociales escolares y una pura igualdad de oportunidades. En efecto, el esquema clásico del análisis de la asignación de lugares en las sociedades modernas - el esquema Origen Educación-Destino- prevé que en el caso de una sociedad puramente meritocrática, los «destinos», es decir, los lugares, derivan de la educación recibida. Entonces, habría fuertes relaciones entre Educación y Destino, lo que se designa aquí como una fuerte influencia.

Es cierto que otras características de los sistemas educativos pueden estar asociadas con los niveles desiguales de influencia de los diplomas. Amarante (2015) muestra que los países con fuerte influencia tienen sistemas educativos más centralizados y jerarquizados que otros, lo que hace que los diplomas sean más visibles para los empleadores, contribuyendo así a su «desempeño». Finalmente, hay una correlación positiva muy marcada entre la influencia de los diplomas y el desempleo juvenil, que refuerza la idea de que la influencia de los diplomas es tanto más necesaria que el «racionamiento» de los empleos; en otras palabras, en un contexto de competencia fuerte por los escasos trabajos, el criterio de los diplomas contribuye a la organización de la fila de espera de los jóvenes. En general, el modo de articulación de los diplomas con las posiciones sociales se inscribe en un conjunto de relaciones específicas para cada país.

\section{LA REPRODUCCIÓN SE JUEGA MÁS DESPUÉS DE LA ESCUELA QUE EN ELLA}

En la sociología de la reproducción, que analiza cómo las posiciones sociales se transmiten de padres a hijos, a la institución escolar se le dio un papel central, particularmente con las tesis de Bourdieu y Passeron (1970). Esto hasta el punto que la función reproductiva de la escuela aparece como una invariante de las sociedades modernas, que necesitan encontrar en los veredictos escolares y en los méritos de las personas, la justificación de sus desigualdades sociales. Estas tesis tenían relación con el débil desarrollo de las encuestas comparativas. Hace veinticinco años, éstas han desarrollado considerablemente y muestran que las relaciones entre las desigualdades escolares 
y las desigualdades sociales están lejos de revestir una cara perfectamente uniforme. Esto lleva a preguntarse si en el mecanismo de reproducción social, la escuela juega el mismo papel en todas partes y con la misma magnitud.

Se pueden seguir dos pistas para comprender estas variaciones. La manera en que se organizan los sistemas escolares causa sin duda la transformación de las desigualdades sociales en desigualdades de logros. También, se puede rastrear una pista menos explorada, sobre la manera cómo los países valoran los diplomas escolares. Las tesis de la reproducción postulan una relación de continuidad con la corriente de la escuela, entre las desigualdades sociales y desigualdades escolares, pero también con el aval entre las desigualdades escolares y las desigualdades de las posiciones sociales, que obtendrán sobre esta base los estudiantes.

Las comparaciones hechas en los estudios recientes demuestran que esta cadena está lejos de ser automática. Para comprender el papel que juega la escuela en la producción y reproducción de las desigualdades sociales no deben interesar solamente las consecuencias escolares de las desigualdades sociales, es necesario también estudiar los efectos sociales de las desigualdades escolares. Porque se imagina fácilmente que las desigualdades escolares de logros y de diplomas jugarán un papel muy diferente, dependiendo de si el país da más o menos peso a los diplomas y desarrolla de manera intensa los sistemas alternativos de calificación profesional y / o de acceso al empleo. Es en el juego de estos dos mecanismos que se arraigan las diferencias entre los países en materia de reproducción social.

Para probar en qué medida la influencia de los diplomas constituye el vector de una reproducción social más o menos pronunciada, es necesario dar un indicador empírico, necesariamente imperfecto, a este concepto muy general. En la literatura económica, se pudo operacionalizar esta variable «reproducción» por el impacto del salario del padre en el de sus hijos (Addio, 2007). Concretamente, se mide la fracción de la diferencia de ingresos existente en un momento dado entre los adultos que se transmite a sus descendientes; cuanto mayor es esta fracción, mayor es la reproducción, en el sentido de la transmisión intergeneracional de los ingresos es fuerte, y la movilidad intergeneracional del ingreso es menor.

La educación juega en esta materia un papel importante en la media en que la transmisión de los ingresos de una generación a otra depende de los rendimientos de la educación: la transmisión padres / hijos de los ingresos es más marcada cuando la educación es muy rentable, cuando la influencia escolar favorece la transformación de las desigualdades escolares en posiciones sociales y, por tanto, cuando las desigualdades incorporadas en los diplomas se convierten en desigualdades sociales de posición.

Se ve entonces que, independientemente de la magnitud de las desigualdades escolares de los logros y de títulos, éstas participan en la reproducción social de diferentes maneras en función del rol que juegan, en los países, los títulos escolares en el acceso a las posiciones sociales. En esta materia, las especificidades nacionales, inscritas en la historia y materializadas en el funcionamiento de la economía y del mercado del trabajo, son obviamente importantes.

\section{DISCUSIÓN Y CONCLUSIONES}

Si bien es probablemente trivial señalar que la educación persigue múltiples finalidades, dar la primacía, incluso la exclusividad, a una de ellas es cuestionable, y la ponderación entre estas finalidades puede ser menos consensual. Las comparaciones internacionales se limitan a comparaciones del rendimiento académico, lo que se puede juzgar cuestionable. Ciertamente, se refiere a la concepción que se tiene del rol de la escuela. Si se considera que los logros son bienes en sí mismos, «las competencias para la vida», es fundamental evaluarlos e identificar los parámetros que los hacen variar, sabiendo que en la materia las comparaciones son indispensables. Una primera observación es que las comparaciones a gran escala pueden polarizarse de hecho en lo que es más fácil de medir. Entonces, se descuidaría la observación de las actitudes, no solamente más difíciles de aprehender sino también quizá menos fácil de comparar de un país a otro. Forjar ciertas actitudes está aún en el corazón de los objetivos educativos, especialmente si se considera la educación como el principal vector de cohesión social. Este tipo de investigación sería aún más valioso ya que las actitudes y el 
rendimiento son relativamente inconexas, con la consecuencia de que algunos países podrían hacer más o menos explícitamente la «elección», privilegiar ciertos objetivos de actitudes más que ciertos objetivos cognitivos, y partir de evaluaciones comparativas basadas únicamente en el rendimiento.

Pero, no se puede ignorar la otra función capital de la escuela que es la asignación a los empleos, en una perspectiva tanto económica (eficacia de este modo de gestión y rentabilidad del capital humano) como sociológica (porque así se fabrica la reproducción social). Y aunque con menor frecuencia, la pregunta ¿en qué momento es legítimo evaluar lo que «produce» la escuela? es crucial: a los 15 años o 10 años después de ingresar a la edad adulta. Entonces, puede ser más la posición social que se ha obtenido que el volumen de los logros escolares lo que importa. Las clasificaciones que resultan de las medidas de los logros a los 15 años son entonces fuertemente relativizados. Se puede alcanzar el mismo nivel de igualdad o desigualdad en la transmisión del estatus social de padres a hijos mediante procesos diferentes del mismo modo que se puede alcanzar niveles comparables de eficacia pedagógica con procesos diferentes. Entonces, no hay un solo modelo y no se puede fundar un diagnóstico en las informaciones aisladas de un contexto (Bottani, 2008), pero es una tendencia posible en las vastas encuestas comparativas, que trabajan variable por variable como si la educación fuera un juego mecánico.

Dicho esto, se acepta focalizar los logros a los 15 años (o en el nivel más alto alcanzado) porque las estadísticas muestran que éstos son para los jóvenes una clave de acceso al empleo. Pero, esta mirada que se polariza en el nivel micro, la de los individuos, no deja de tener efectos adversos, porque lo que vale para las personas no siempre vale en el nivel macro, el del país. En el nivel de las personas, la fuerte influencia de los diplomas es interesante para los que poseen más diplomas, aunque penalice a los "vencidos» del sistema escolar. Pero a nivel macro, ella causa una carrera hacia adelante (Duru-Bellat, 2006) y la rigidez de la sociedad, ya que la reproducción social es más marcada. Ésta dirige el modelo meritocrático, aunque es difícil predecir si, cuando la influencia de los diplomas es más moderada, la sociedad es entonces menos injusta, ya que la escuela no tiene el monopolio de clasificar a los individuos, como lo quiere la ideología meritocrática, que solo acepta las desigualdades producidas por el diploma y rechaza como ilegítimas aquellas que pasarían por las cualidades poco escolares.

\section{REFERENCIAS BIBLIOGRÁFICAS}

Addio, D. (2007). A. International Transmission of Disadvantage: Mobility or immobility across generations? OECD Social Employment and Migration Working Papers, (52), pp. 150-164.

Amarante, V. (2015). La medición de la desigualdad: otros indicadores. Santiago, Chile: CEPAL. Goldthorpe, J. H. (2014). El papel de la educación en la movilidad social intergeneracional: problemas de la investigación empírica en sociología y algunos indicadores teóricos de la economía. Sage journals, (26), pp. 265-289.

Bottani, N. (2008). Le niveau d'huile, le moteur et la voiture : les enjeux d'une évaluation de la qualité de l'enseignement par les indicateurs. Education et formations, (78), pp. 9-23.

Bourdieu, P. y Passeron, J-C. (1970). La Reproduction. Paris, France: Ed. de Minuit.

Demeuse, M. y Baye, A. (2008). Indicateurs d'équité éducative. Une analyse de la ségrégation académique et sociale. Revue Française de Pédagogie, (165), pp. 91-103.

Dubet, F., Duru-Bellat, M. y Veretout, A. (2010). Les sociétés et leur école. Emprise du diplôme et cohésion sociale. Paris, France: Seuil.

Duru-Bellat, M. (2006). L'inflation scolaire. Les désillusions de la méritocratie. Paris, France: Seuil.

Duru-Bellat, M., Mons, N. y Bydanova, E. (2008). Cohésion scolaire et politiques éducatives. Revue Française de Pédagogie, (164), pp. 37-54. 
Goldthorpe, J.H. (2014). Problems of Meritocracy. IN R. Erikson \& J. Jonsson (eds.). Can Education Be Equalized? (pp. 255287). Boulder, United States: Westview Press.

Kerckoff, A.C. (1995). Institutional Arrangements and Stratification Processes in Industrial Societies. Annual Review of Sociology, (15), pp. 323-347.

Shavit, Y. y Müller, W. (1998). From school to work. Oxford, England: Clarendon Press. 